UAB-FT-588

October 2005

\title{
The scalar and pseudoscalar sector in a five-dimensional approach to chiral symmetry breaking
}

\author{
Leandro Da Rold and Alex Pomarol \\ IFAE, Universitat Autònoma de Barcelona, 08193 Bellaterra, Barcelona
}

\begin{abstract}
We study the scalar and pseudoscalar sector in a five-dimenional model describing chiral symmetry breaking. We calculate the scalar and pseudoscalar two-point correlator, the mass spectrum and interactions. We also obtain the scalar and pseudoscalar contributions to the coefficients of the chiral lagrangian and determine the scalar form factor of the pseudo-Goldstone bosons. Most quantities show a good agreement with QCD.
\end{abstract}




\section{Introduction}

The AdS/CFT correspondence [1] has provided a new approach to tackle strongly coupled theories. This has recently boosted the search for string realizations of QCD-like theories $[2,3]$. A crucial ingredient in these realizations is a (compact) warped extra dimension that plays the role of the energy scale in the QCD-like theory. It is therefore interesting to look for properties of QCD in the strong regime that can be derived from weakly coupled theories in five-dimensions. Examples of this type of properties have already been found in high-energy hadron scattering [4], string breaking [5], hadron form factors and hadron spectroscopy [6]-[13].

In Refs. [10, 11] a five-dimensional model was proposed to study the breaking of the chiral symmetry in QCD. The model was described in terms of infinite weakly coupled resonances, similar to QCD in the large- $N_{c}$ limit. The vector sector was studied and several relations among couplings and masses were derived based only on the (warped) five-dimensionality of the space. The predictions of the model showed a good agreement with QCD.

Here we will extend this analysis to the scalar and pseudoscalar sector. We will calculate the scalar and pseudoscalar two-point correlator and show that they have a similar behavior to that in QCD. We will obtain the masses and couplings of the resonances, pointing out the implications of working with a warped extra dimension. We will also calculate the scalar form factor of the pseudo-Goldstone boson (PGB) and the pseudo(scalar) contributions to the $L_{i}$ coefficients of the low-energy chiral lagrangian. A prediction for the quark masses will also be given. We will compare our results with the QCD experimental data whenever this is available.

\section{A five-dimensional model for QCD}

The 5D model proposed to study the properties of QCD with 3 flavors consists in a theory where the chiral symmetry $\mathrm{U}(3)_{L} \otimes \mathrm{U}(3)_{R}$ is gauged in the $5 \mathrm{D}$ bulk ${ }^{1}$. Parity is defined as the interchange $L \leftrightarrow R$. The bulk fields are the gauge bosons $L_{M}, R_{M}$ and a complex scalar field $\Phi$ transforming as a $\left(\mathbf{3}_{\mathbf{L}}, \overline{\mathbf{3}}_{\mathbf{R}}\right)$. This scalar plays the role of the operator $q \bar{q}$ in QCD whose vacuum expectation value $(\mathrm{VEV})$ is responsible for the breaking of the chiral symmetry. The 5D metric in conformal coordinates is defined as

$$
d s^{2}=a^{2}(z)\left(\eta_{\mu \nu} d x^{\mu} d x^{\nu}-d z^{2}\right)
$$

where $a$ is the warp factor. We will work within $\mathrm{AdS}_{5}$

$$
a(z)=\frac{L}{z}
$$

where $L$ is the AdS curvature radius. The $\mathrm{AdS}_{5}$ metric will guarantee conformal invariance of the model at high energies. The fifth dimension is assumed to be compact, $L_{0} \leq z \leq L_{1}$ [14].

\footnotetext{
${ }^{1}$ The $\mathrm{U}(1)_{A}$ is broken by the anomaly that, although it will not be studied here, can also be incorporated in extra-dimensional models along the lines of Ref. [3].
} 
The boundary at $z=L_{1}$ generates a mass gap in the model (breaks the conformal symmetry at energies $\left.\sim 1 / L_{1}\right)$, while the boundary at $z=L_{0}$ is only needed to regulate the theory. When performing calculations one must take the limit $L_{0} \rightarrow 0$ and eliminate the divergences that one encounters by properly adding UV-boundary counterterms [1]. The action is given by

$$
S_{5}=\int d^{4} x \int d z \mathcal{L}_{5}
$$

where

$$
\mathcal{L}_{5}=\sqrt{g} M_{5} \operatorname{Tr}\left[-\frac{1}{4} L_{M N} L^{M N}-\frac{1}{4} R_{M N} R^{M N}+\frac{1}{2}\left|D_{M} \Phi\right|^{2}-\frac{1}{2} M_{\Phi}^{2}|\Phi|^{2}\right] .
$$

The covariant derivative is defined as

$$
D_{M} \Phi=\partial_{M} \Phi+i L_{M} \Phi-i \Phi R_{M}
$$

where $M=(\mu, 5)$ and $\Phi=\mathbb{1} / \sqrt{3} \Phi_{s}+\Phi_{a} T_{a}$, with $\operatorname{Tr}\left[T_{a} T_{b}\right]=\delta_{a b}$ (and similarly for $L_{M}$ and $\left.R_{M}\right)$. For the value of the scalar mass we take $M_{\Phi}^{2}=-3 / L^{2}$ that, by the AdS/CFT dictionary, corresponds to associate the scalar $\Phi$ with a CFT operator of dimension 3 such as $\bar{q} q$. Solving the equation of motion for $\Phi$ we obtain

$$
\langle\Phi\rangle \equiv v(z)=c_{1} z+c_{2} z^{3}
$$

where $c_{1}$ and $c_{2}$ can be written in terms of the value of $v$ at the boundaries

$$
c_{1}=\frac{\widetilde{M}_{q} L_{1}^{3}-\xi L_{0}^{2}}{L L_{1}\left(L_{1}^{2}-L_{0}^{2}\right)}, \quad c_{2}=\frac{\xi-\widetilde{M}_{q} L_{1}}{L L_{1}\left(L_{1}^{2}-L_{0}^{2}\right)},
$$

where we have defined

$$
\left.\widetilde{M}_{q} \equiv \frac{L}{L_{0}} v\right|_{L_{0}},\left.\quad \xi \equiv L v\right|_{L_{1}}
$$

It can be shown that a nonzero $\widetilde{M}_{q}$ corresponds to an explicit breaking of the chiral symmetry in the UV, while a nonzero $c_{2}$ corresponds to a spontaneous breaking of the chiral symmetry in the IR. Therefore the value of $c_{2}$ is determined dynamically by minimizing the action. In order to get a nonzero value for $c_{2}$ in the chiral limit $\left(\widetilde{M}_{q}=0\right)$ we add a potential for $\Phi$ on the IR-boundary:

$$
\mathcal{L}_{\mathrm{IR}}=-\left.a^{4} V(\Phi)\right|_{L_{1}}, \quad V(\Phi)=-\frac{1}{2} m_{b}^{2} \operatorname{Tr}|\Phi|^{2}+\lambda \operatorname{Tr}|\Phi|^{4}
$$

An origin for this type of potentials can be found in string constructions [2, 3]. To determine the value of $c_{2}$, or equivalently the value of $\xi$, we must minimize the effective 4D action obtained after substituting Eq. (6) into the $5 \mathrm{D}$ action. For $L_{0} \rightarrow 0$, this is given by

$$
S_{\mathrm{eff}} \simeq-\int d^{4} x \operatorname{Tr}\left\{M_{5} L\left[\frac{-\widetilde{M}_{q}^{2}}{2 L_{0}^{2}}+\frac{\widetilde{M}_{q}^{2}}{L_{1}^{2}}-2 \frac{\xi \widetilde{M}_{q}}{L_{1}^{3}}+\frac{3}{2} \frac{\xi^{2}}{L_{1}^{4}}\right]+V(\xi) \frac{L^{4}}{L_{1}^{4}}\right\}
$$

that is minimized for

$$
\xi^{2}=\frac{1}{4 \lambda}\left(m_{b}^{2} L^{2}-3 M_{5} L\right)+\mathcal{O}\left(\widetilde{M}_{q}\right)
$$


This 5D model depends on 5 parameters: ${ }^{2} \widetilde{M}_{q}, M_{5}, L_{1}, \xi$ and $\lambda$. The value of $\widetilde{M}_{q}$ is related to the quark masses as we will see below. The values of $M_{5}, L_{1}$ and $\xi$ were determined in Ref. [11] from the gauge sector of the theory. By using the QCD values for $N_{c}, M_{\rho}$ and $M_{a_{1}}$, it was found [11]:

$$
M_{5} L=\frac{N_{c}}{12 \pi^{2}} \equiv \tilde{N}_{c}, \quad \frac{1}{L_{1}} \simeq 320 \mathrm{MeV}, \quad \xi \simeq 4 .
$$

Our predictions will be given using the above values (although in certain cases we will study the dependence of the predictions on $\xi$ ). This leaves the scalar sector of the theory depending only on one parameter, $\lambda$. An estimate of its value can be obtained using naive dimensional analysis $(\mathrm{NDA})$ that gives $\lambda \sim 1 /\left(16 \pi^{2}\right) \sim 10^{-2}-10^{-3}$.

\section{The scalar and pseudoscalar sector}

We define

$$
\Phi=(v+S) e^{i P / v}
$$

where $S$ corresponds to a real scalar and $P$ to a real pseudoscalar under parity. Since we will be considering the chiral limit $\widetilde{M}_{q} \rightarrow 0$, we have $v \propto \mathbb{1}$ and the symmetry breaking pattern $\mathrm{U}(3)_{L} \otimes \mathrm{U}(3)_{R} \rightarrow \mathrm{U}(3)_{V}$. Under $\mathrm{SU}(3)_{V}$ we have that both $S$ and $P$ transform as $\mathbf{1}+\mathbf{8}$. We will work in the unitary gauge. This corresponds to add the gauge fixing terms

$$
\begin{aligned}
& \mathcal{L}_{G F}^{V}=-\frac{M_{5} a}{2 \xi_{V}} \operatorname{Tr}\left[\partial_{\mu} V_{\mu}-\frac{\xi_{V}}{a} \partial_{5}\left(a V_{5}\right)\right]^{2}, \\
& \mathcal{L}_{G F}^{A}=-\frac{M_{5} a}{2 \xi_{A}} \operatorname{Tr}\left[\partial_{\mu} A_{\mu}-\frac{\xi_{A}}{a} \partial_{5}\left(a A_{5}\right)-\xi_{A} \sqrt{2} a^{2} v P\right]^{2},
\end{aligned}
$$

where $V_{M}, A_{M}=\frac{1}{\sqrt{2}}\left(L_{M} \pm R_{M}\right)$, and take the limit $\xi_{V, A} \rightarrow \infty$, i.e.

$$
\partial_{5}\left(a V_{5}\right)=0, \quad P=-\frac{1}{\sqrt{2} a^{3} v} \partial_{5}\left(a A_{5}\right)
$$

The above equation will allow us to write $P$ as a function of $A_{5}$ in the 5D lagrangian. After integration by parts, the 5D quadratic terms for the scalar $S$ and the pseudoscalar $A_{5}$ are given by

$$
\begin{aligned}
& \mathcal{L}_{S}=-\frac{a^{3} M_{5}}{2} \operatorname{Tr}\left\{S\left[\partial^{2}-a^{-3} \partial_{5} a^{3} \partial_{5}+a^{2} M_{\Phi}^{2}\right] S\right\}, \\
& \mathcal{L}_{A_{5}}=-\frac{a M_{5}}{2} \operatorname{Tr}\left\{A_{5}\left[\partial^{2} \mathcal{D}+\mathcal{D}\left(2 v^{2} a^{2} \mathcal{D}\right)\right] A_{5}\right\},
\end{aligned}
$$

where $\mathcal{D}$ is a differential operator defined by

$$
\mathcal{D}=1-\partial_{5}\left(\frac{1}{2 v^{2} a^{3}} \partial_{5} a\right) .
$$

\footnotetext{
${ }^{2}$ We trade $m_{b}^{2}$ for $\xi$ by means of Eq. (11). In the following we will take $\xi \rightarrow \xi \mathbb{1}+\mathcal{O}\left(\widetilde{M}_{q}\right)$ and treat $\xi$ as a parameter.
} 
The scalar and pseudoscalar field has also 4D boundary terms that, after using the 5D equation of motion for $A_{5}$ (i.e., $\left.\mathcal{D} A_{5}=-\partial^{2} A_{5} /\left(2 v^{2} a^{2}\right)\right)$ and Eq. (11), can be written as ${ }^{3}$

$$
\begin{aligned}
\mathcal{L}_{\text {bound }} & =-\left.\frac{M_{5} a}{2} \operatorname{Tr}\left[a^{2} S \partial_{5} S+A_{5} \frac{\partial^{2}}{2 v^{2} a^{3}} \partial_{5}\left(a A_{5}\right)+2 A_{\mu} \partial_{\mu} A_{5}\right]\right|_{L_{0}} ^{L_{1}}-\left.a^{4} V(S)\right|_{L_{1}} \\
& +\left.M_{5} a^{3} \operatorname{Tr}[S] \partial_{5} v\right|_{L_{0}},
\end{aligned}
$$

where

$$
\left.V(S)\right|_{L_{1}}=\left.m_{S}^{2} \operatorname{Tr}\left[S^{2}\right]\right|_{L_{1}}+\mathcal{O}\left(S^{3}\right), \quad m_{S}^{2}=\frac{4 \lambda \xi^{2}}{L^{2}}-\frac{3 M_{5}}{2 L}+\mathcal{O}\left(\widetilde{M}_{q}\right) .
$$

To cancel the quadratic terms on the IR-boundary of Eq. (18) we impose the conditions

$$
\left.\left[M_{5} \partial_{5}+2 a m_{S}^{2}\right] S\right|_{L_{1}}=0,\left.\quad A_{5}\right|_{L_{1}}=0
$$

The boundary conditions on the UV-boundary will be specified later.

The interactions between scalars and pseudoscalars that we will be considering are

$$
\begin{aligned}
\mathcal{L}_{S A_{5} A_{5}} & =\frac{a^{3} M_{5}}{2} \operatorname{Tr}\left[\frac{S}{v^{3} a^{6}}\left(\partial_{\mu} \partial_{5}\left(a A_{5}\right)\right)^{2}-4 v S\left(\mathcal{D} A_{5}\right)^{2}\right] \\
\mathcal{L}_{A_{5}^{4}} & =\frac{M_{5}}{96 a^{9} v^{6}} \operatorname{Tr}\left[\left(\partial_{5}\left(a A_{5}\right) \overleftrightarrow{\partial_{\mu}} \partial_{5}\left(a A_{5}\right)\right)^{2}\right] .
\end{aligned}
$$

The $S V V$ interaction is absent. This is a consequence of the $\mathrm{U}(3)_{V}$ invariance and the fact that only dimension-four operators are considered in Eq. (4). This interaction, however, could be induced by higher-dimensional operators or loop effects.

With the above lagrangian for the scalar and pseudoscalar sector we can calculate any relevant physical quantity. We will be considering two approximations. First, we will be working at the tree-level. According to Eq. (12) this corresponds to work in the large- $N_{c}$ limit. Since loop effects are expected to be of order $1 / N_{c}$, our predictions for QCD quantities will have a $30 \%$ uncertainty. Second, we will take the chiral limit $\widetilde{M}_{q} \rightarrow 0$. For the pseudoscalar sector this limit will be taken in the following way. We will first perform the calculations with $c_{1} \rightarrow 0$ and fixed $L_{0}$ (this is equivalent to $\widetilde{M}_{q} \rightarrow \xi L_{0}^{2} / L_{1}^{3}$ and $c_{2} \rightarrow \xi /\left(L L_{1}^{3}\right)$ ). Next we will take the limit $L_{0} \rightarrow 0$. This procedure simplifies the calculations and avoids singularities at $z=L_{0}$.

\subsection{The scalar and pseudoscalar correlator}

In this section we will calculate the scalar and pseudoscalar two-point correlator. In QCD these are defined as

$$
\Pi_{S, P}\left(p^{2}\right)=-\int d^{4} x e^{i p x}\left\langle J_{S, P}(x) J_{S, P}(0)\right\rangle,
$$

\footnotetext{
${ }^{3}$ One obtains the same result if, instead of the equation of motion, one uses the mass eigenfunction equation, $\mathcal{D} A_{5}=m^{2} A_{5} /\left(2 v^{2} a^{2}\right)$, as we will do later to perform a Kaluza-Klein decomposition of the sector.
} 
where $J_{S}=\bar{q} q$ and $J_{P}=i \bar{q} \gamma_{5} q$. The correlators $\Pi_{S, P}$ can be obtained from the generating functional $\mathcal{S}$ according to

$$
\Pi_{S}=\frac{\delta^{2} \mathcal{S}}{\delta s^{2}}, \quad \Pi_{P}=\frac{\delta^{2} \mathcal{S}}{\delta p_{s}^{2}},
$$

where $s$ and $p_{s}$ are the scalar and pseudoscalar external sources coupled to QCD:

$$
\mathcal{L}=-\operatorname{Tr}\left[\bar{q}_{L} \phi q_{R}\right]+\text { h.c. }, \quad \phi=M_{q}+s+i p_{s} .
$$

The AdS/CFT correspondence tells us [1] that $\mathcal{S}$ is obtained in the 5D theory by integrating out the bulk fields restricted to a given UV-boundary value. These boundary values play the role of the external sources coupled to QCD. In particular, for the 5D scalar field we have

$$
\left.\Phi\right|_{L_{0}}=\alpha \frac{L_{0}}{L} \phi
$$

where the constant $\alpha$ will be determined by matching with the QCD correlators in the UV as we will see later. Up to the quadratic order in the fields, Eq. (26) leads to

$$
\left.S\right|_{L_{0}}=\alpha \frac{L_{0}}{L}\left(s+\alpha \frac{p_{s}^{2}}{2 \widetilde{M}_{q}}\right),\left.\quad P\right|_{L_{0}}=-\left.\frac{\partial_{5}\left(a A_{5}\right)}{\sqrt{2} a^{3} v}\right|_{L_{0}}=\alpha \frac{L_{0}}{L} p_{s}
$$

Let us calculate $\mathcal{S}=\int d^{4} x \mathcal{L}_{\text {eff }}$ at the quadratic level for $S$ and $A_{5}$. By solving the equations of motion from Eq. (16) with the boundary conditions of Eqs. (20) and (27), and substituting the solution back into the action, we get (in momentum space) ${ }^{4}$

$$
\mathcal{L}_{\text {eff }}=\frac{1}{2} \Pi_{S}\left(p^{2}\right) \operatorname{Tr}\left[s^{2}\right]+\frac{1}{2} \Pi_{P}\left(p^{2}\right) \operatorname{Tr}\left[p_{s}^{2}\right]+\Gamma_{S} \operatorname{Tr}[s] .
$$

For a $\mathrm{AdS}_{5}$ space $\Pi_{S}$ can be given analytically at the tree-level. We obtain

$$
\Pi_{S}\left(p^{2}\right)=\alpha^{2} M_{5} L\left[\frac{1}{L_{0}^{2}}+\frac{i p}{L_{0}} \frac{J_{0}\left(i p L_{0}\right)+b(p) Y_{0}\left(i p L_{0}\right)}{J_{1}\left(i p L_{0}\right)+b(p) Y_{1}\left(i p L_{0}\right)}\right],
$$

where $J_{n}, Y_{n}$ are Bessel functions, $p$ is the Euclidean momentum and $b(p)$ is determined by the IR-boundary condition of Eq. (20):

$$
b(p)=-\frac{i p L_{1} J_{2}\left(i p L_{1}\right)-\frac{8 \lambda \xi^{2}}{M_{5} L} J_{1}\left(i p L_{1}\right)}{i p L_{1} Y_{2}\left(i p L_{1}\right)-\frac{8 \lambda \xi^{2}}{M_{5} L} Y_{1}\left(i p L_{1}\right)}
$$

Taking the limit $L_{0} \rightarrow 0$ we find

$$
\Pi_{S}\left(p^{2}\right) \simeq \alpha^{2} M_{5} L\left[\frac{1}{L_{0}^{2}}+\frac{1}{2} p^{2} \ln \left(p^{2} L_{0}^{2}\right)+\frac{\pi p^{2}}{2 b(p)}\right] .
$$

\footnotetext{
${ }^{4}$ There is also a mixing term between $p_{s}$ and the longitudinal part of $\left.A_{\mu}\right|_{L_{0}}$ that we are not writing.
} 
The divergent terms for $L_{0} \rightarrow 0$ can be absorbed in a bare mass and a bare kinetic term for $s$. After this renormalization the correlator is finite. For large momentum $p L_{1} \gg 1$, we find

$$
\Pi_{S}\left(p^{2}\right) \simeq \frac{\alpha^{2} M_{5} L}{2} p^{2} \ln p^{2}
$$

as expected from the conformal symmetry. Matching with QCD in which at large momentum we have

we obtain, using Eq. (12),

$$
\Pi_{S}^{\mathrm{QCD}}\left(p^{2}\right) \simeq \frac{N_{c}}{8 \pi^{2}} p^{2} \ln p^{2}
$$

$$
\alpha=\sqrt{3} .
$$

The next to leading terms in the large momentum expansion in Eq. (32) are suppressed exponentially, contrary to QCD where one finds that the scalar correlator has power corrections. This is because we assumed, for simplicity, that the scalar had a potential only on the IR-boundary. In more realistic models such as those arising from string theories the scalar potential is present in the 5D bulk (although peaked towards the IR). In these cases the scalar correlator has power corrections. Also, if the 5D metric deviate in the IR from AdS or if higher-dimensional operators are included in Eq.(4), then power corrections can be present in $\Pi_{S}$.

For small momentum $\Pi_{S}\left(p^{2}\right)$ can be approximated by

$$
\Pi_{S}\left(p^{2}\right) \simeq 3 \tilde{N}_{c}\left[-\frac{2}{L_{1}^{2}}+\frac{\tilde{N}_{c}}{2 \lambda \xi^{2} L_{1}^{2}}\right]+\mathcal{O}\left(p^{2}\right) .
$$

The scalar correlator Eq. (29) can also be written as a sum over infinitely narrow resonances, similarly as in large- $N_{c}$ QCD:

$$
\Pi_{S}\left(p^{2}\right)=\sum_{n} \frac{F_{S_{n}}^{2} M_{S_{n}}^{2}}{p^{2}+M_{S_{n}}^{2}}
$$

Therefore the masses of the scalar resonances can be determined by finding the poles of Eq. (31), i.e., by the equation $b(p)=0$. In Fig. 1 we plot the value of the mass of the first and second scalar resonance as a function of $\lambda$ for $\xi=4$. The first resonance mass ranges from $M_{S_{1}}=0$ $\mathrm{MeV}(\lambda \rightarrow 0)$ to $M_{S_{1}}=1226 \mathrm{MeV}(\lambda \rightarrow \infty)$. We compare this value with the masses of the $a_{0}$ states (since these are the QCD scalars whose masses are not very sensitive to $M_{q}$ ). We see that for a value of $\lambda$ close to its NDA estimate, $\lambda \sim 10^{-2}-10^{-3}$, the mass of the first scalar resonance is closer to that of $a_{0}(980)$ than to that of $a_{0}(1450)$. Nevertheless we must recall that we are working in the large- $N_{c}$ limit and then corrections can be as large as $30 \%$. Consequently we cannot discard to associate $S^{(1)}$ with $a_{0}(1450)$. The scalar decay constants $F_{S_{n}}$ are determined by the residues of $\Pi_{S}$. We obtain

$$
F_{S_{n}}^{2}=\frac{3 \tilde{N}_{c} \pi M_{S_{n}}^{2}\left(\frac{8 \lambda \xi^{2}}{M_{5} L} Y_{1}\left(M_{S_{n}} L_{1}\right)-M_{S_{n}} L_{1} Y_{2}\left(M_{S_{n}} L_{1}\right)\right)}{M_{S_{n}} L_{1}\left(1-\frac{8 \lambda \xi^{2}}{M_{5} L}\right) J_{0}\left(M_{S_{n}} L_{1}\right)+\left(\frac{8 \lambda \xi^{2}}{M_{5} L}+M_{S_{n}}^{2} L_{1}^{2}-2\right) J_{1}\left(M_{S_{n}} L_{1}\right)}
$$

For $\lambda \simeq 10^{-3}$ we obtain $M_{S_{1}} \simeq 1 \mathrm{GeV}$ and $F_{S_{1}} \simeq 260 \mathrm{MeV}$, while for the second resonance we get $M_{S_{2}} \simeq 1900 \mathrm{MeV}$ and $F_{S_{2}} \simeq 370 \mathrm{MeV}$. Using this result we can calculate the value of the 


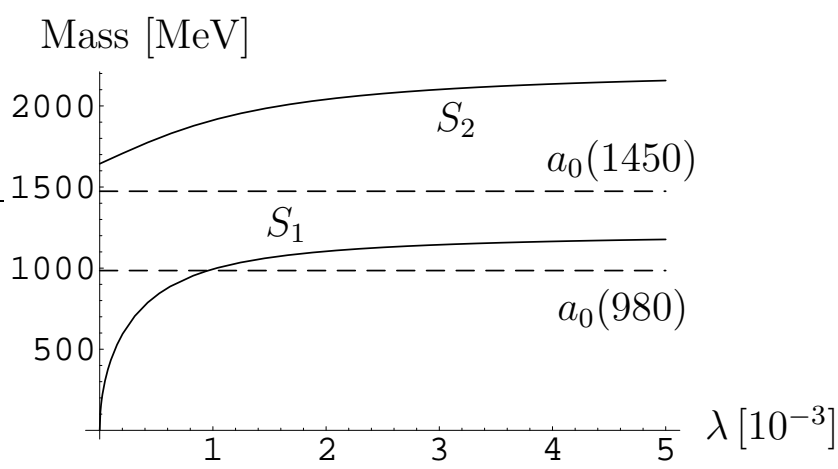

Figure 1: Mass of the first and second scalar resonances as a function of $\lambda$ for $\xi=4$. The dashed lines show the experimental values for the masses of the scalar resonances $a_{0}(980)$ and $a_{0}(1450)$.

coupling $c_{m}$ defined in Ref. [15]. We obtain $c_{m}=F_{S_{1}} M_{S_{1}} /\left(4 B_{0}\right) \simeq 41 \mathrm{MeV}$ (taking the value of $B_{0}$ from Eq. (63)) very close to the value used in Ref. [15]: $c_{m} \simeq 42 \mathrm{MeV}$.

To calculate the pseudoscalar correlator $\Pi_{P}$ we must rely on numerical analysis. Only for small and large momentum we are able to give analytical results. For large momentum $p L_{1} \gg 1$ we have

$$
\Pi_{P}\left(p^{2}\right)=\frac{3 \tilde{N}_{c}}{L_{0}^{2}}+p^{2}\left[\frac{3 \tilde{N}_{c}}{2} \ln \left(p^{2} L_{0}^{2}\right)-\frac{c_{6}^{P}}{p^{6}}+\mathcal{O}\left(\frac{1}{p^{12}}\right)\right], \quad \text { where } \quad c_{6}^{P}=-\frac{64}{5} \frac{3 \tilde{N}_{c} \xi^{2}}{L_{1}^{6}} .
$$

Again the divergences can be cancelled by adding a proper mass and a kinetic term for the pseudoscalar $p_{s}$ on the UV-boundary. From Eqs. (32) and (38) we can obtain the correlator $\Pi_{S P}=\Pi_{S}-\Pi_{P}$ at large momentum. It drops as $\Pi_{S P} \sim c_{6}^{P} / p^{4}$. Comparing with $\Pi_{L R}=$ $\Pi_{V}-\Pi_{A} \sim c_{6} / p^{4}$, we find $c_{6}^{P}=12 c_{6}[11]$ in strong disagreement with QCD in which one has $c_{6}^{P}=3 c_{6}$. This can be improved if, as we said, we consider more realistic theories where the scalar potential is present in the $5 \mathrm{D}$ bulk and therefore $\Pi_{S}$ has power corrections.

At low momentum ${ }^{5}$ and for $\xi \gg 1$ we find

$$
\Pi_{P}\left(p^{2}\right) \simeq \frac{2 \widetilde{B}_{0}^{2} F_{\pi}^{2}}{p^{2}}-\tilde{N}_{c} \widetilde{B}_{0}^{2}+\mathcal{O}\left(p^{2}\right)
$$

where

$$
\widetilde{B}_{0}=\frac{2 \sqrt{3} \tilde{N}_{c} \xi}{F_{\pi}^{2} L_{1}^{3}}, \quad F_{\pi}^{2}=\Pi_{A}(0) \stackrel{\xi \gg 1}{\simeq} \frac{2^{5 / 3} \pi \tilde{N}_{c}}{3^{1 / 6} \Gamma\left(\frac{1}{3}\right)^{2}} \frac{\xi^{2 / 3}}{L_{1}^{2}} .
$$

$\Pi_{A}\left(p^{2}\right)$ is the axial-vector correlator calculated in Ref. [11]. The first term of Eq. (39) shows a pole at $p^{2}=0$ as expected due to the presence of the massless PGB.

By looking at the poles of $\Pi_{P}$ we can find the pseudoscalar masses. The lowest mode is the massless PGB of the spontaneous chiral symmetry breaking. There is a nonet of PGBs but we

\footnotetext{
${ }^{5}$ In order to obtain the correct result it is important to take the limit $L_{0} \rightarrow 0$ before taking $p^{2} \rightarrow 0[16]$.
} 


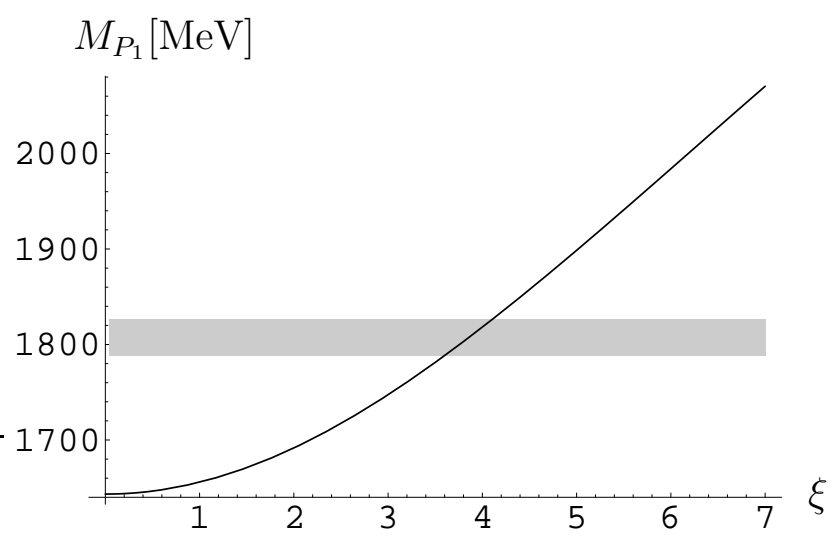

Figure 2: Mass of the first massive pseudoscalar resonance as a function of $\xi$. The shadow band shows the experimental value for $\pi(1800)$.

must recall that the inclusion of the $\mathrm{U}(1)_{A}$-anomaly will give mass to the singlet [3]. The mass of the first massive resonance is shown in Fig. 2 as a function of $\xi$. We see that its value is far from the mass of the $\pi(1300)$ state. Nevertheless, we find that, for $\xi \simeq 4, M_{P_{1}}$ is close to the mass of $\pi(1800)$ suggesting that this could be the state to be associated with our first massive pseudoscalar resonance. For this resonance we find a decay constant $F_{P_{1}} \simeq 374 \mathrm{MeV}$.

Finally, we calculate the linear term in Eq. (28) to be associated in QCD with the $\bar{q} q$ condensate: $\Gamma_{S}=-\left\langle J_{S}\right\rangle$. We find

$$
\Gamma_{S}=\sqrt{3} \tilde{N}_{c} \frac{\widetilde{M}_{q} L_{1}^{2}+2 \xi L_{0}^{2} / L_{1}-3 \widetilde{M}_{q} L_{0}^{2}}{L_{0}^{2}\left(L_{1}^{2}-L_{0}^{2}\right)} \quad \stackrel{\widetilde{M}_{q} \rightarrow 0}{\longrightarrow} \quad \frac{2 \sqrt{3} \tilde{N}_{c} \xi}{L_{1}\left(L_{1}^{2}-L_{0}^{2}\right)} \quad \stackrel{L_{0} \rightarrow 0}{\longrightarrow} \quad \frac{2 \sqrt{3} \tilde{N}_{c} \xi}{L_{1}^{3}} .
$$

\subsection{Scalar meson interactions}

To study the interactions it is convenient to perform a Kaluza-Klein (KK) decomposition of the 5D fields:

$$
S(x, z)=\frac{1}{\sqrt{M_{5} L}} \sum_{n=1}^{\infty} f_{n}^{S}(z) S^{(n)}(x), \quad A_{5}(x, z)=\frac{1}{\sqrt{M_{5} L}} \sum_{n=0}^{\infty} f_{n}^{P}(z) P^{(n)}(x) .
$$

We impose the following boundary conditions on the UV-boundary:

$$
\left.S\right|_{L_{0}}=0,\left.\left.\quad P\right|_{L_{0}} \propto \partial_{5}\left(a A_{5}\right)\right|_{L_{0}}=0,
$$

that cancel the boundary terms of Eq. (18). The wave-functions of the KK-modes $S^{(n)}$ are given by

$$
f_{n}^{S}(z)=\frac{z^{2}}{N_{S_{n}} L_{1}^{2}}\left[J_{1}\left(M_{S_{n}} z\right)-\frac{J_{1}\left(M_{S_{n}} L_{0}\right)}{Y_{1}\left(M_{S_{n}} L_{0}\right)} Y_{1}\left(M_{S_{n}} z\right)\right] \quad \stackrel{L_{0} \rightarrow 0}{\longrightarrow} \quad \frac{z^{2}}{N_{S_{n}} L_{1}^{2}} J_{1}\left(M_{S_{n}} z\right),
$$

where $N_{S_{n}}$ is a constant fixed by canonically normalizing the fields, $\int a^{3}\left(f_{n}^{S}\right)^{2} d z / L=1$. In Fig. 3 we plot the wave-functions of the first two KK-modes. 


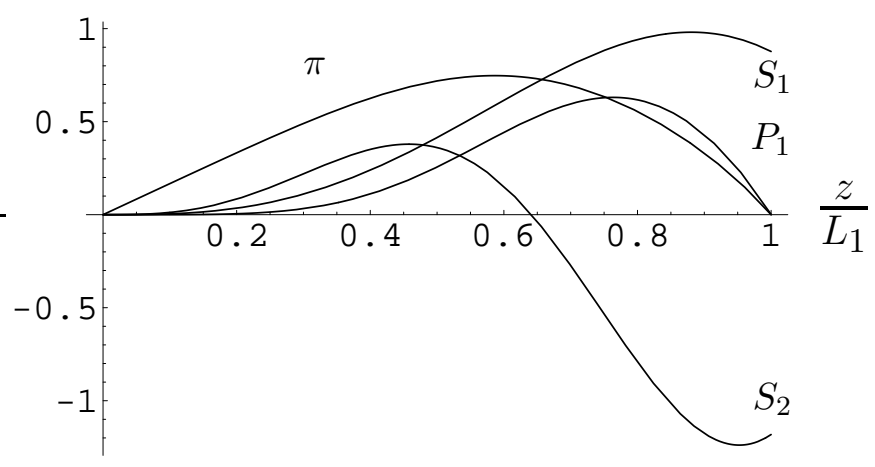

Figure 3: Wave-functions of the $n=1,2$ scalar resonance, the PGB and the first massive pseudoscalar for $\xi=4$ and $\lambda=10^{-3}$.

The equation that determines the wave-functions of the pseudoscalars can be obtained from Eq. (16). This is given by

$$
\mathcal{D} f_{n}^{P}=\frac{M_{P_{n}}^{2}}{2 v^{2} a^{2}} f_{n}^{P} .
$$

The lowest state, $P^{(0)} \equiv \pi$, is the PGB that in the limit $L_{0} \rightarrow 0$ is massless. Its wave-function is given by

$$
f^{\pi}(z) \stackrel{L_{0} \rightarrow 0}{\longrightarrow} \frac{z^{3}}{L_{1}^{3} N_{0}}\left[I_{2 / 3}\left(\frac{\sqrt{2} \xi}{3} \frac{z^{3}}{L_{1}^{3}}\right)-\frac{I_{2 / 3}(\sqrt{2} \xi / 3)}{K_{2 / 3}(\sqrt{2} \xi / 3)} K_{2 / 3}\left(\frac{\sqrt{2} \xi}{3} \frac{z^{3}}{L_{1}^{3}}\right)\right]
$$

where $N_{0}$ is determined by the condition $-\left.\frac{1}{2 a^{2} v^{2} L} f^{\pi} \partial_{5}\left(a f^{\pi}\right)\right|_{L_{0}}=1$. The wave-function of the massive modes must be obtained numerically from Eq. (45) with the normalization condition $\int d z\left(f_{n}^{P} M_{P_{n}}\right)^{2} /\left(2 v^{2} a L\right)=1$. The wave-functions of $\pi$ and $P^{(1)}$ are shown in Fig. 3.

The couplings between the resonances are easily obtained by integrating the 5D interactions over $z$ with the corresponding wave-functions. The coupling of a scalar to two PGBs comes from Eq. (21). We obtain

$$
\mathcal{L}_{S_{n} \pi \pi}=G_{n \pi \pi} \operatorname{Tr}\left[S^{(n)}\left(\partial_{\mu} \pi\right)^{2}\right]
$$

where $G_{n \pi \pi}$ is given by

$$
G_{n \pi \pi}=\frac{1}{\sqrt{M_{5} L^{3}}} \int d z f_{n}^{S} \frac{\left[\partial_{5}\left(a f^{\pi}\right)\right]^{2}}{2 a^{3} v^{3}} .
$$

In Fig. 4 we show the coupling of the first modes as a function of $\lambda$ for $\xi=3,4$. We find that $G_{n \pi \pi}$ becomes smaller as $n$ increases. This property is also present in the coupling between a vector resonance and two PGBs, and it is due to the oscillatory behaviour of the KK wavefunctions. Associating $S^{(1)}$ with $a_{0}(980)$, we find that $M_{S_{1}} \simeq 980 \mathrm{MeV}$ for $\lambda \simeq 10^{-3}$, and the prediction of the $5 \mathrm{D}$ model for the $a_{0} \pi \eta$ coupling is $G_{1 \pi \pi} \simeq 5.4 \mathrm{GeV}^{-1}$ for $\xi=4$. In the notation of Ref. [15] we find $c_{d}=F_{\pi}^{2} G_{1 \pi \pi} / 2 \simeq 20 \mathrm{MeV}$ to be compared to the value $\left|c_{d}\right| \simeq 32$ $\mathrm{MeV}$ given there. If the width of $a_{0}(980)$ is dominated by the decay to $\eta \pi$ we find

$$
\Gamma\left(a_{0} \rightarrow \eta \pi\right) \simeq 27-56 \mathrm{MeV}, \text { for } \xi=4-3 .
$$




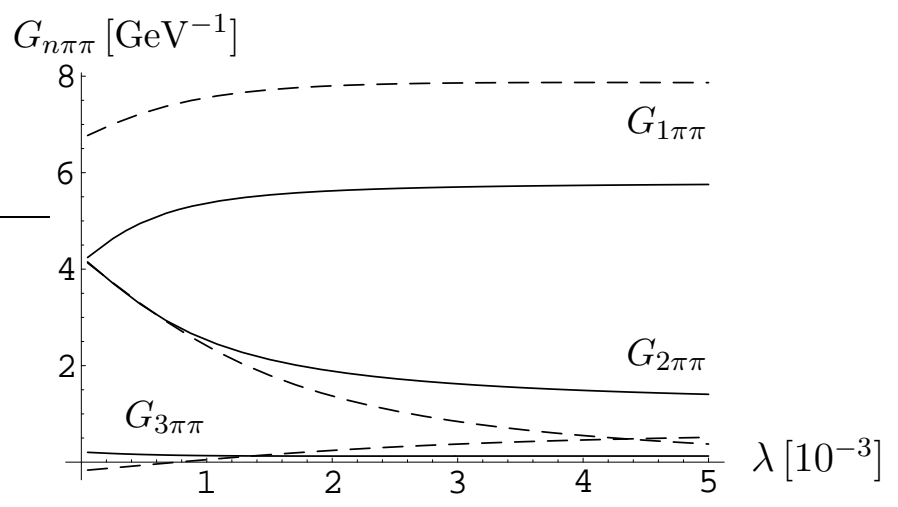

Figure 4: Coupling of the $n=1,2,3$ scalar resonance to two PGBs as a function of $\lambda$ for $\xi=4$ (solid line) and $\xi=3$ (dashed line).

Unfortunately, the experimental value of the width of $a_{0}(980)$ has a large uncertainty $\Gamma\left(a_{0}\right)=$ $50-100 \mathrm{MeV}[17]$.

\section{4 (Pseudo)Scalar contributions to PGB interactions}

By integrating the heavy scalar resonances we obtain the following four-PGB interaction

$$
\mathcal{L}_{\pi^{4}}^{(8)}=\frac{1}{2}\left\{\operatorname{Tr}\left[\left(\partial_{\mu} \pi\right)^{2}\left(\partial_{\nu} \pi\right)^{2}\right]-\frac{1}{3} \operatorname{Tr}^{2}\left[\left(\partial_{\mu} \pi\right)^{2}\right]\right\} \sum_{n} \frac{G_{n \pi \pi}^{2}}{p^{2}+M_{S_{n}}^{2}},
$$

from the scalar octet and

$$
\mathcal{L}_{\pi^{4}}^{(1)}=\frac{1}{6} \operatorname{Tr}^{2}\left[\left(\partial_{\mu} \pi\right)^{2}\right] \sum_{n} \frac{G_{n \pi \pi}^{2}}{p^{2}+M_{S_{n}}^{2}},
$$

from the scalar singlet. The sum over the KK-modes in Eqs. (50) and (51) is dominated by the first resonance. At large momentum we find that the first resonance gives $82 \%$ of the total contribution and this percentage rises to $94 \%$ at zero momentum (for $\lambda \simeq 10^{-3}$ ). Therefore, as in the vector case [11], we find that the scalar mediation of the four-PGB interaction is dominated by the exchange of the first resonance.

Four-PGB interactions can also arise from Eq. (22). We find

$$
\mathcal{L}_{\pi^{4}}=\frac{g_{\pi^{4}}}{4} \operatorname{Tr}\left[\left(\pi \overleftrightarrow{\partial_{\mu}} \pi\right)^{2}\right], \text { where } \quad g_{\pi^{4}}=\frac{1}{24 M_{5} L^{2}} \int d z \frac{\left[\partial_{5}\left(a f^{\pi}\right)\right]^{4}}{a^{9} v^{6}}
$$

At high energies the four-PGB amplitude arising from Eq. (52) grows as $\sim E^{2}$. Nevertheless, this bad energy behavior of the four-PGB amplitude is cured by the contribution arising from Eqs. (50) and (51) that cancels the $E^{2}$ terms. This occurs thanks to the sum rule

$$
\sum_{n} G_{n \pi \pi}^{2}=6 g_{\pi^{4}}
$$


Eq. (53) is a property of any 5D model in which the breaking of the chiral symmetry is realized by the Higgs mechanism.

We can also calculate the coupling of the PGB to the source $s$ that defines the scalar form factor of the PGB. Apart from a contact piece given by

$$
\mathcal{L}_{\pi^{2} s}=-\widetilde{B}_{0} \operatorname{Tr}\left[\pi^{2} s\right]
$$

this coupling is mediated by the octet and singlet scalar resonances that gives respectively

$$
\begin{aligned}
\mathcal{L}_{\pi^{2} s}^{(8)} & =\left\{\operatorname{Tr}\left[\left(\partial_{\mu} \pi\right)^{2} s\right]-\frac{1}{3} \operatorname{Tr}\left[\left(\partial_{\mu} \pi\right)^{2}\right] \operatorname{Tr}[s]\right\} \sum_{n} \frac{G_{n \pi \pi} F_{S_{n}} M_{S_{n}}}{p^{2}+M_{S_{n}}^{2}} \\
\mathcal{L}_{\pi^{2} s}^{(1)} & =\frac{1}{3} \operatorname{Tr}\left[\left(\partial_{\mu} \pi\right)^{2}\right] \operatorname{Tr}[s] \sum_{n} \frac{G_{n \pi \pi} F_{S_{n}} M_{S_{n}}}{p^{2}+M_{S_{n}}^{2}} .
\end{aligned}
$$

The scalar form factor of the PGB is then given by (normalized to unity at zero momentum)

$$
\mathcal{F}_{\pi}^{S}(p)=1-\frac{p^{2}}{2 \widetilde{B}_{0}} \sum_{n} \frac{G_{n \pi \pi} F_{S_{n}} M_{S_{n}}}{p^{2}+M_{S_{n}}^{2}} .
$$

At low momentum the sum in Eq. (56) is dominated by the first resonance that gives $75 \%$ of the total contribution (for $\lambda \simeq 10^{-3}$ ). At large momentum we find that the form factor goes as $1 / p^{2}$, as expected from the conformal symmetry [4]. The cancellation of the constant term in $\mathcal{F}_{\pi}^{S}(p)$ occurs due to the sum rule

$$
\sum_{n} G_{n \pi \pi} F_{S_{n}} M_{S_{n}}=2 \widetilde{B}_{0}
$$

This sum rule is fulfilled in any $5 \mathrm{D}$ model whose metric approaches to $\mathrm{AdS}_{5}$ for $z \rightarrow 0$ (conformal theories in the UV). In Eq. (57) we find that the first two resonances give a similar contribution, while the contributions of the heavier resonances tend to cancel out. Therefore we see that $\mathcal{F}_{\pi}^{S}(p)$ is very well approximated by the exchange of only the first two resonances.

\subsection{The Chiral Lagrangian}

At energies below the massive resonances our 5D model is described by the QCD chiral lagrangian. In this section we calculate the (pseudo)scalar contributions to the coefficients of the chiral lagrangian and compare these results with the QCD values.

Up to $\mathcal{O}\left(p^{2}\right)$, the chiral lagrangian for the octet of PGB, $\pi=\pi_{a} T_{a}$, is given by [18]

$$
\mathcal{L}_{2}=\frac{F_{\pi}^{2}}{4} \operatorname{Tr}\left[D_{\mu} U^{\dagger} D^{\mu} U+U^{\dagger} \chi+\chi^{\dagger} U\right]
$$

where

$$
D_{\mu} U=\partial_{\mu} U-i R_{\mu} U+i U L_{\mu}, \quad U=e^{i \sqrt{2} \pi / F_{\pi}}
$$


and

$$
\chi=2 B_{0}\left(M_{q}+s+i p_{s}\right), \quad M_{q}=\operatorname{Diag}\left(m_{u}, m_{d}, m_{s}\right) .
$$

The prediction of our model for $F_{\pi}$ is given in Eq. (40). It gives

$$
F_{\pi} \simeq 87\left(\frac{\xi}{4}\right)^{\frac{1}{3}} \mathrm{MeV}
$$

For the prediction of $B_{0}$ we can use Eq. (41):

$$
\langle\bar{q} q\rangle=-F_{\pi}^{2} B_{0}=-2 \sqrt{3} \tilde{N}_{c} \frac{\xi}{L_{1}^{3}} \simeq-(226 \mathrm{MeV})^{3}\left(\frac{\xi}{4}\right)
$$

that leads to

$$
B_{0}=\frac{2 \sqrt{3} \tilde{N}_{c} \xi}{F_{\pi}^{2} L_{1}^{3}} \simeq 1520\left(\frac{\xi}{4}\right)^{\frac{1}{3}} \mathrm{MeV}
$$

Notice that $B_{0}=\widetilde{B}_{0}$ as it should be, since the first term of Eq. (39) can also be deduced by integrating out the PGB at tree-level in the chiral lagrangian. The relation $B_{0}=\widetilde{B}_{0}$ also leads to the right matching of Eq. (54) with the chiral lagrangian. The value of the quark masses $M_{q}$ is related to the VEV of $\Phi$ on the UV-boundary. Using Eqs. (8), (26) and (34) we obtain ${ }^{6}$

$$
M_{q}=\frac{1}{\sqrt{3}} \widetilde{M}_{q}
$$

From the chiral lagrangian we have

$$
\left(m_{\pi}^{2}\right)_{a b}=2 B_{0} \operatorname{Tr}\left[M_{q} T_{a} T_{b}\right],
$$

that for $m_{\pi^{0}} \simeq 135 \mathrm{MeV}$ and $m_{K^{0}} \simeq 498 \mathrm{MeV}$ gives

$$
m_{u}+m_{d}=11.5 \mathrm{MeV}, \quad m_{s}=150 \mathrm{MeV} .
$$

The value of the quark masses in Eq. (66) are scale independent. This is because we took $M_{\Phi}^{2}=-3 / L^{2}$ that corresponds, by the AdS/CFT dictionary, to fix the dimension of $M_{q}$ to be exactly one. In QCD however the quark masses evolve with the energy scale $\mu$. To minimize this discrepancy we must compare our predictions with the experimental values of the quark masses taken at the lowest energy scale $(\sim 1 \mathrm{GeV})$. From Ref. [17] we have $m_{u}+m_{d}=7-16$ $\mathrm{MeV}$ and $m_{s}=108-175 \mathrm{MeV}$ at $\mu \sim 1 \mathrm{GeV}$ in good agreement with Eq. (66).

At $\mathcal{O}\left(p^{4}\right)$ the chiral lagrangian is given by [18]

$$
\begin{aligned}
\mathcal{L}_{4} & =L_{1} \operatorname{Tr}^{2}\left[D_{\mu} U^{\dagger} D^{\mu} U\right]+L_{2} \operatorname{Tr}\left[D_{\mu} U^{\dagger} D_{\nu} U\right] \operatorname{Tr}\left[D^{\mu} U^{\dagger} D^{\nu} U\right]+L_{3} \operatorname{Tr}\left[D_{\mu} U^{\dagger} D^{\mu} U D_{\nu} U^{\dagger} D^{\nu} U\right] \\
& +L_{4} \operatorname{Tr}\left[D_{\mu} U^{\dagger} D^{\mu} U\right] \operatorname{Tr}\left[U^{\dagger} \chi+\chi^{\dagger} U\right]+L_{5} \operatorname{Tr}\left[D_{\mu} U^{\dagger} D^{\mu} U\left(U^{\dagger} \chi+\chi^{\dagger} U\right)\right] \\
& +L_{6} \operatorname{Tr}^{2}\left[U^{\dagger} \chi+\chi^{\dagger} U\right]+L_{7} \operatorname{Tr}^{2}\left[U^{\dagger} \chi-\chi^{\dagger} U\right]+L_{8} \operatorname{Tr}\left[\chi^{\dagger} U \chi^{\dagger} U+U^{\dagger} \chi U^{\dagger} \chi\right] \\
& -i L_{9} \operatorname{Tr}\left[F_{R}^{\mu \nu} D_{\mu} U D_{\nu} U^{\dagger}+F_{L}^{\mu \nu} D_{\mu} U^{\dagger} D_{\nu} U\right]+L_{10} \operatorname{Tr}\left[U^{\dagger} F_{R}^{\mu \nu} U F_{L \mu \nu}\right] .
\end{aligned}
$$

\footnotetext{
${ }^{6}$ In Refs. [10, 11] the quark masses did not have the correct normalization since the value of $\alpha$ was not calculated.
} 
At tree-level, the (pseudo)scalar resonances only contribute ${ }^{7}$ to $L_{1,3,4,5,6,8}$. The contributions to the coefficients $L_{1}$ and $L_{3}$ coming from the octet and singlet scalar can be read from Eqs. (50) and (51). We obtain

$$
\begin{aligned}
& L_{1}^{(8)}=-\frac{1}{3} L_{3}^{(8)}, \quad L_{1}^{(1)}=-L_{1}^{(8)}, \\
& L_{3}^{(8)}=\sum_{n} \frac{G_{n \pi \pi}^{2} F_{\pi}^{4}}{8 M_{S_{n}}^{2}}, \quad L_{3}^{(1)}=0 .
\end{aligned}
$$

The octet and singlet contribution to the coefficient $L_{1}$ cancels out, as expected from large- $N_{c}$ [18], and only $L_{3}$ gets a nonzero scalar contribution. For $\lambda \simeq 10^{-3}$ and $\xi=4$ (3) we obtain $L_{3}^{(8)} \simeq 0.2 \cdot 10^{-3}\left(0.3 \cdot 10^{-3}\right)$. Adding the vector contribution to $L_{3}$ calculated in Ref. [11] we get $L_{3} \simeq-2.4 \cdot 10^{-3}\left(-1.7 \cdot 10^{-3}\right)$ to be compared with the experimental value [19] $L_{3}^{\exp } \simeq-3.5 \pm 1.1$. The scalar contribution to $L_{4}$ and $L_{5}$ can be obtained from Eq. (55):

$$
\begin{aligned}
L_{4}^{(8)} & =-\frac{1}{3} L_{5}^{(8)}, \quad L_{4}^{(1)}=-L_{4}^{(8)}, \\
L_{5}^{(8)} & =\frac{F_{\pi}^{2}}{8 B_{0}} \sum_{n} \frac{G_{n \pi \pi} F_{S_{n}}}{M_{S_{n}}}, \quad L_{5}^{(1)}=0 .
\end{aligned}
$$

As expected from large- $N_{c}$, the total contribution to $L_{4}$ is zero. The value of $L_{5}$ is shown in Fig. 5 as a function of $M_{S_{1}}$ for $\xi=3,4$. For $M_{S_{1}} \sim 1 \mathrm{GeV}$ we obtain $L_{5} \simeq 1.1 \cdot 10^{-3}$ in good agreement with experiments. $L_{5}$ can also be calculated from the axial-vector correlator [11]:

$$
L_{5}=\left.\frac{1}{16 B_{0}} \frac{d \Pi_{A}}{d M_{q}}\right|_{M_{q}=0}
$$

For $\xi \gg 1$ with $\lambda \xi^{2}$ fixed $^{8}$, we obtain

$$
L_{5} \simeq \frac{\tilde{N}_{c} \pi^{3}}{\sqrt{3} \Gamma\left(\frac{1}{3}\right)^{6}}\left[1-\frac{2 \tilde{N}_{c}}{3 F_{\pi}^{2} L_{1}^{2}}\right]+\frac{F_{\pi}^{4} L_{1}^{4}}{192 \lambda \xi^{4}} \simeq 1.2 \cdot 10^{-3}\left[1-0.23\left(\frac{4}{\xi}\right)^{\frac{2}{3}}+0.09\left(\frac{10^{-3}}{\lambda}\right)\left(\frac{4}{\xi}\right)^{\frac{8}{3}}\right] .
$$

Finally, the coefficient $L_{6,8}$ can be computed from the correlators $\Pi_{S, P}$. We have

$$
\begin{aligned}
& L_{6}^{(8)}=-\frac{1}{3} L_{8}^{(8)}, \quad L_{6}^{(1)}=-L_{6}^{(8)} \\
& L_{8}^{(8)}=\left.\frac{1}{32 B_{0}^{2}} \frac{d}{d p^{2}}\left[p^{2}\left(\Pi_{S}\left(p^{2}\right)-\Pi_{P}\left(p^{2}\right)\right)\right]\right|_{p^{2}=0}, \quad L_{8}^{(1)}=0 .
\end{aligned}
$$

\footnotetext{
${ }^{7} L_{7}$ will not be studied here since it arises from integrating out the singlet PGB that becomes massive when the $\mathrm{U}(1)_{A}$ anomaly is considered.

${ }^{8}$ In Ref. [11] the value of $L_{5}$ was given for $\lambda \xi^{2} \gg 1$ and therefore the last term of Eq. (73) was not present. This last term arises due to the $\xi$ dependence on $\widetilde{M}_{q}$ - see Eq. (11). Also a factor $1 / 2$ was missing in Eq. (67) of Ref. [11] and therefore the prediction of $L_{5}$ given there was a factor 2 larger.
} 

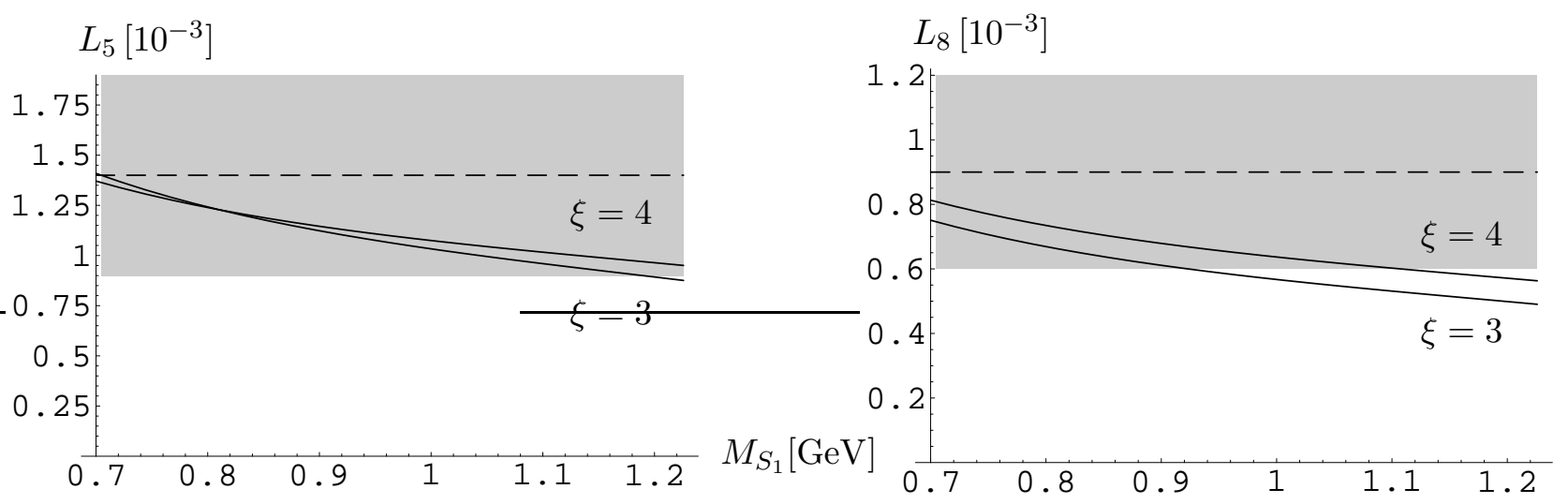

Figure 5: Prediction for $L_{5}$ and $L_{8}$ as a function of $M_{S_{1}}$. The horizontal line corresponds to the experimental value with the error bands [19].

Then $L_{6}=L_{6}^{(8)}+L_{6}^{(1)}=0$, as expected from large- $N_{c}$. Using Eqs. (35), (39) and (63) in the above equation, we obtain

$$
L_{8} \simeq \frac{\tilde{N}_{c}}{32}\left[1-\frac{6}{B_{0}^{2} L_{1}^{2}}+\frac{3 \tilde{N}_{c}}{2 \lambda \xi^{2} B_{0}^{2} L_{1}^{2}}\right] \simeq 8 \cdot 10^{-4}\left[1-0.27\left(\frac{4}{\xi}\right)^{\frac{2}{3}}+0.11\left(\frac{10^{-3}}{\lambda}\right)\left(\frac{4}{\xi}\right)^{\frac{8}{3}}\right] .
$$

Notice that this expression is only valid for $\xi \gg 1$ with $\lambda \xi^{2}$ fixed. In Fig. 5 we show the exact value of $L_{8}$ as a function of $M_{S_{1}}$. For $M_{S_{1}} \simeq 1 \mathrm{GeV}$ and $\xi=4$ we obtain $L_{8} \simeq 0.6 \cdot 10^{-3}$ again in good agreement with the experimental value. From Fig. 5 one can see that small values of $M_{S_{1}}$ are preferred. The coefficient $L_{8}$ can also be written as

$$
L_{8}=\frac{1}{32 B_{0}^{2}}\left[F_{S_{1}}^{2}+\sum_{n=1}^{\infty}\left(F_{S_{n+1}}^{2}-F_{P_{n}}^{2}\right)\right],
$$

that shows that in the limit where the chiral symmetry is restored, $\xi \rightarrow 0$ and $F_{S_{n+1}} \rightarrow F_{P_{n}}$, only the first term remains. For $\xi \simeq 4$ we find that the first term still dominates (it gives $70 \%$ of the total contribution for $\lambda \simeq 10^{-3}$ ) since the other resonances, being so heavy, are not very sensitive to chiral symmetry breaking.

\section{Conclusions}

We have analyzed the scalar and pseudoscalar sector of a five-dimensional model proposed to study mesons in QCD. We have calculated the scalar and pseudoscalar two-point correlator and we have obtained the mass spectrum and interactions. This has allowed us to determine the (pseudo)scalar contribution to the scalar form factor of the PGB as well as the contribution to the $L_{i}$ coefficients of the chiral lagrangian. We have also found two interesting sum rules for the scalar couplings and masses of the resonances that are fulfilled generically in $\mathrm{AdS}_{5}$ models. 
Comparing with the experimental data, we have found a good agreement for the $L_{i}$ predictions (see Fig. 5) and the quark masses. For the first massive pseudoscalar resonance we have obtained a mass around $1800 \mathrm{MeV}$, quite different from the mass of the lowest QCD pseudoscalar resonance $\pi(1300)$. This has suggested us to associate this state to $\pi(1800)$. We have also given predictions for the scalar couplings and decay constants but the absence of clean experimental data has not allowed us to compare them with QCD.

Previous approaches to calculate the scalar and pseudoscalar spectrum and/or determine their contribution to $L_{i}$ can be found in Refs. [20]-[23]. In particular, the analysis of Refs. [21, $22]$ has certain similarity with ours. Refs. [21, 22] work in the large- $N_{c}$ limit where QCD is described as a theory of infinite hadron resonances. These sets of infinite hadrons, however, are approximated in Refs. [21, 22] by taking only the lowest modes, and their masses and couplings are determined by demanding a good high-energy behaviour of the correlators and form factors. In our approach we have shown that the correlators and form factors have the correct highenergy behaviour since this is dictated by the conformal symmetry. We have also found that, in certain cases, it can be a good approximation to take only the lowest resonance. Therefore in these cases our approach and that of Refs. [21, 22] give similar results. Nevertheless, we have showed that the single-resonance approximation is not always justified (for example in Eq. (57)) and this approximation can lead to large errors in the determination of the scalar parameters.

The analysis carried out here can be extended to study three-point or four-point correlation functions or to incorporate the effects of $m_{s}$ either in the mass spectrum or in the interactions. Also the effects of higher-dimensional operators or departures from $\mathrm{AdS}_{5}$ in the IR-boundary can be studied. These effects are important to study the power corrections in the correlators. We leave this analysis for the future.

\section{Acknowledgements}

We would like to thank Rafel Escribano, Matthias Jamin, Santi Peris and Eduard de Rafael for very useful discussions. This work was partly supported by the MCyT and FEDER Research Project FPA2002-00748 and DURSI Research Project 2001-SGR-00188. The work of LD was supported by the Spanish Education Office (MECD) under an FPU scholarship.

\section{References}

[1] J. M. Maldacena, Adv. Theor. Math. Phys. 2 (1998) 231; S. S. Gubser, I. R. Klebanov and A. M. Polyakov, Phys. Lett. B 428 (1998) 105; E. Witten, Adv. Theor. Math. Phys. 2 (1998) 253.

[2] For certain realizations of string theories describing flavor see: A. Karch and E. Katz, JHEP 0206 (2002) 043; M. Kruczenski, D. Mateos, R. C. Myers and D. J. Winters, JHEP 
0307, 049 (2003); T. Sakai and J. Sonnenschein, JHEP 0309, 047 (2003); C. Nunez, A. Paredes and A. V. Ramallo, JHEP 0312 (2003) 024; J. Babington, J. Erdmenger, N. J. Evans, Z. Guralnik and I. Kirsch, Phys. Rev. D 69 (2004) 066007; N. J. Evans and J. P. Shock, Phys. Rev. D 70 (2004) 046002; K. Ghoroku and M. Yahiro, Phys. Lett. B 604, 235 (2004); K. Suzuki, arXiv:hep-th/0411076; S. Kuperstein, JHEP 0503 (2005) 014; D. Bak and H. U. Yee, Phys. Rev. D 71, 046003 (2005); A. Paredes and P. Talavera, Nucl. Phys. B 713, 438 (2005); I. Kirsch and D. Vaman, Phys. Rev. D 72 (2005) 026007; R. Apreda, J. Erdmenger and N. Evans, arXiv:hep-th/0509219.

[3] T. Sakai and S. Sugimoto, Prog. Theor. Phys. 113 (2005) 843; arXiv:hep-th/0507073.

[4] J. Polchinski and M. J. Strassler, Phys. Rev. Lett. 88 (2002) 031601; JHEP 0305, 012 (2003); H. Boschi-Filho and N. R. F. Braga, Phys. Lett. B 560 (2003) 232.

[5] A. Karch, E. Katz and N. Weiner, Phys. Rev. Lett. 90, 091601 (2003).

[6] H. Boschi-Filho and N. R. F. Braga, JHEP 0305 (2003) 009.

[7] D. T. Son and M. A. Stephanov, Phys. Rev. D 69 (2004) 065020.

[8] S. Hong, S. Yoon and M. J. Strassler, JHEP 0404 (2004) 046; arXiv:hep-th/0409118; arXiv:hep-ph/0501197.

[9] S. J. Brodsky and G. F. de Teramond, Phys. Lett. B 582 (2004) 211; Phys. Rev. Lett. 94, 201601 (2005); arXiv:hep-ph/0509269.

[10] J. Erlich, E. Katz, D. T. Son and M. A. Stephanov, arXiv:hep-ph/0501128.

[11] L. Da Rold and A. Pomarol, Nucl. Phys. B 721 (2005) 79.

[12] J. Hirn and V. Sanz, arXiv:hep-ph/0507049.

[13] N. Evans, J. P. Shock and T. Waterson, Phys. Lett. B 622 (2005) 165.

[14] L. Randall and R. Sundrum, Phys. Rev. Lett. 83 (1999) 3370.

[15] G. Ecker, J. Gasser, A. Pich and E. de Rafael, Nucl. Phys. B 321 (1989) 311.

[16] R. Contino and A. Pomarol, JHEP 0411 (2004) 058.

[17] S. Eidelman et al. [Particle Data Group Collaboration], Phys. Lett. B 592 (2004) 1.

[18] J. Gasser and H. Leutwyler, Nucl. Phys. B 250 (1985) 465.

[19] A. Pich, arXiv:hep-ph/9806303.

[20] S. Peris, M. Perrottet and E. de Rafael, JHEP 9805 (1998) 011.

[21] M. F. L. Golterman and S. Peris, Phys. Rev. D 61 (2000) 034018.

[22] M. Jamin, J. A. Oller and A. Pich, Nucl. Phys. B 622 (2002) 279.

[23] A. Bramon, R. Escribano and J. L. L. Martinez, Phys. Rev. D 69 (2004) 074008. 